\title{
Optimal antenna currents
}

\author{
M. Gustafsson*
}

\begin{abstract}
Antenna current optimization offers performance bounds and suggestions for the distribution of the desired antenna currents. Many current optimization problems can be formulated as convex optimization problems that are solved efficiently with explicit error bounds. The formulations as convex optimization problems are also illuminating as they can easily be generalized to specific antenna problems by adding convex constraints.
\end{abstract}

\section{INTRODUCTION}

Optimization is used in antenna design to improve existing antenna designs and to find new designs [1]. Random search algorithms, such as genetic algorithms [2] and particle swarm, and gradient based algorithms dominate the field due to the complexity of the antenna design problems. Optimization of the antenna current density $[3,4]$ is inherently different from antenna optimization and can often be formulated as convex optimization problems [4]. The formulations as convex optimization problems are advantageous as there are many efficient solvers and the solvers can provide explicit error estimates [5]. We combine quadratic forms, such as stored energy and radiated power, linear forms, such as near- and far fields and induced currents, and norms to formulate convex optimization problems relevant for specific antenna problems [4].

The evaluation of the stored energy $[6,7]$ matrices are in principle already implemented in many Method of Moments (MoM) codes based on Galerkins method [7]. It is sufficient to separate the electric and magnetic parts of the MoM impedance matrix from the Electric Field Integral Equation (EFIE) and to add a non-singular part. Here, we restrict the analysis to surface currents in free space. We present an overview of antenna current optimization.

\section{CURRENT OPTIMIZATION}

Optimization is a powerful tool in antenna design $[1,2]$ that can handle the contradictory requirements on performance and size. Here, we consider generic antenna geometries to illustrate the approach. The antenna structure is denoted $V$ and consists of an antenna region $V_{1} \subset V$ and a remainder $V-V_{1}$. The antenna is part of the structure

\footnotetext{
*Department of Electrical and Information Technology, Lund University, Box 118, SE-221 00 Lund, Sweden. e-mail: mats.gustafsson@eit.lth.se, tel.: +46462227506.
}

but restricted to the region $V_{1}$. We assume that the antenna designer is free to determine the material properties in the region $V_{1}$ but not allowed to alter the material properties of the remaining region $V-V_{1}$.

We can consider many optimization problems for the antenna design. We use the Q-factor to estimate the fractional bandwidth and the case with a minimal Q-factor can be written

$$
\text { minimize } \frac{\text { stored energy }}{\text { radiated power }}=\frac{\max \left\{W_{\mathrm{e}}, W_{\mathrm{m}}\right\}}{P_{\mathrm{rad}}}
$$

for lossless antennas, where the Q-factor is obtained by multiplication with $2 \omega$ and $\omega$ denotes the angular frequency. We minimize the $Q$ for an antenna by changing the material properties in the region $V_{1}$ for fixed material properties in $V-V_{1}$, see e.g., [8,9]. The minimal Q-factor (1) can be reformulated as a minimization problem for the stored energy subject to a fixed radiated power $P_{\mathrm{r}}$, i.e.,

$$
\begin{array}{ll}
\text { minimize } & \text { stored energy }=\max \left\{W_{\mathrm{e}}, W_{\mathrm{m}}\right\} \\
\text { subject to } & \text { radiated power }=P_{\mathrm{r}}
\end{array}
$$

The two formulations (1) and (2) are equivalent but the latter formulation is more powerful for current optimization as it is easy to generalize it by including additional constraints.

The classical problem of maximal gain over $Q$, $G / Q$ is

$$
\text { minimize } \frac{\text { stored energy }}{\text { partial radiation intensity }}
$$

where $G$ is the partial gain and (3) can be written

$$
\text { minimize stored energy }=\max \left\{W_{\mathrm{e}}, W_{\mathrm{m}}\right\}
$$$$
\text { subject to partial radiation intensity }=P_{0}
$$

\section{CONVEX OPTIMIZATION}

Convex optimization problems are solved with efficient algorithms [5] and dual problems are used for posterior error estimates. One can often state that a problem is solved if it is formulated as a convex optimization problem. Convex optimization includes linear programming (LP), quadratic programing (QP), and quadratically constrained quadratic programing (QCQP) as special cases, see also Fig. 1 for an illustration of convex functions. 


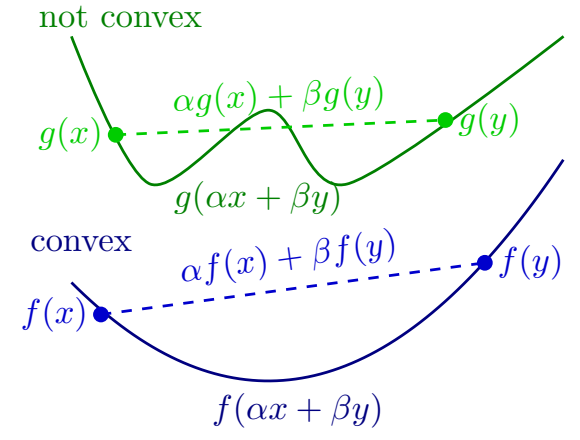

Figure 1: Illustration of convex and non-convex functions. Convex functions satisfy $f(\alpha \mathbf{x}+\beta \mathbf{y}) \leq$ $\alpha f(\mathbf{x})+\beta f(\mathbf{y})$ for $\alpha, \beta \geq 0$, i.e., the curve is below the straight line between two points [5].

We first expand the current density in basis function that implies the approximations $W_{\mathrm{e}} \approx \mathbf{J}^{\mathrm{H}} \mathbf{X}_{\mathrm{e}} \mathbf{J}$ and $W_{\mathrm{m}} \approx \mathbf{J}^{\mathrm{H}} \mathbf{X}_{\mathrm{m}} \mathbf{J}$ of the stored electric and magnetic energies, respectively. The gain Q-factor quotient (3) is

$$
\frac{G(\hat{\boldsymbol{k}}, \hat{\boldsymbol{e}})}{Q} \approx \frac{4 \pi|\mathbf{F} \mathbf{J}|^{2}}{\eta_{0} \max \left\{\mathbf{J}^{\mathrm{H}} \mathbf{X}_{\mathrm{e}} \mathbf{J}, \mathbf{J}^{\mathrm{H}} \mathbf{X}_{\mathrm{m}} \mathbf{J}\right\}}
$$

where $\mathbf{F J}$ is the MoM approximation of the farfield in the direction $\hat{\boldsymbol{k}}$ projected on $\hat{\boldsymbol{e}}$. Using the scaling invariance of $G / Q$ in the current density $\mathbf{J}$, i.e., $G / Q$ is invariant for the complex scaling $\mathbf{J} \rightarrow \alpha \mathbf{J}$, we rewrite the maximization of $G / Q$ into minimization of the stored energy for a fixed partial radiation intensity (4)

$$
\begin{array}{ll}
\operatorname{minimize} \mathbf{J} & \max \left\{\mathbf{J}^{\mathrm{H}} \mathbf{X}_{\mathrm{e}} \mathbf{J}, \mathbf{J}^{\mathrm{H}} \mathbf{X}_{\mathrm{m}} \mathbf{J}\right\} \\
\text { subject to } & |\mathbf{F} \mathbf{J}|^{2}=1,
\end{array}
$$

where we for simplicity use the dimensionless normalization $|\mathbf{F J}|^{2}=1$ that can be written $|\mathbf{F J}|=1$. Moreover, the scaling invariance shows that we can consider an arbitrary phase $\operatorname{Re}\{\mathbf{F J}\}=1$ that removes the absolute value [4]. In total, we have the convex optimization problem to minimization of the stored energy for a fixed partial far-field in one direction [4], i.e.,

$$
\begin{array}{ll}
\operatorname{minimize}_{\mathbf{J}} & \max \left\{\mathbf{J}^{\mathrm{H}} \mathbf{X}_{\mathrm{e}} \mathbf{J}, \mathbf{J}^{\mathrm{H}} \mathbf{X}_{\mathrm{m}} \mathbf{J}\right\} \\
\text { subject to } & \operatorname{Re}\{\mathbf{F J}\}=1 .
\end{array}
$$

Let $\mathbf{J}_{\mathrm{o}}$ denote a current density column matrix that solves (7). The minimal value of the stored energy in (7) is unique. The solution $\mathbf{J}_{\mathrm{o}}$ gives an upper bound on $G / Q$ for the considered direction $\hat{\boldsymbol{k}}$ and polarization $\hat{e}$, i.e.,

$$
\frac{G(\hat{\boldsymbol{k}}, \hat{\boldsymbol{e}})}{Q} \leq\left.\frac{G(\hat{\boldsymbol{k}}, \hat{\boldsymbol{e}})}{Q}\right|_{\mathrm{ub}}=\frac{4 \pi\left|\mathbf{F} \mathbf{J}_{\mathrm{o}}\right|^{2}}{\eta_{0} \max \left\{\mathbf{J}_{\mathrm{o}}^{\mathrm{H}} \mathbf{X}_{\mathrm{e}} \mathbf{J}_{\mathrm{o}}, \mathbf{J}_{\mathrm{o}}^{\mathrm{H}} \mathbf{X}_{\mathrm{m}} \mathbf{J}_{\mathrm{o}}\right\}}
$$

The convex optimization problem (7) can be written in many alternative forms. Introduce the stored energy $W$ and use that $W_{\mathrm{e}} \leq W$ and $W_{\mathrm{m}} \leq$ $W$ to get the alternative convex optimization problem

$$
\begin{array}{cl}
\operatorname{minimize}_{\mathbf{J}} & W \\
\text { subject to } & \mathbf{J}^{\mathrm{H}} \mathbf{X}_{\mathrm{e}} \mathbf{J} \leq W \\
& \mathbf{J}^{\mathrm{H}} \mathbf{X}_{\mathrm{m}} \mathbf{J} \leq W \\
& \operatorname{Re}\{\mathbf{F J}\}=1,
\end{array}
$$

where we used a normalized stored energy $W$. There are several efficient implementations that solve convex optimization problems, here we use CVX [10], that gives the MATLAB code

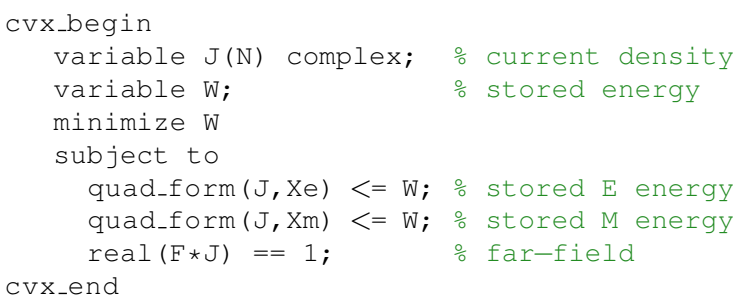

CVX solves the convex optimization problem iteratively, see the CVX manual [10] for details.

\section{CONCLUSIONS}

Current optimization can be considered as an alternative antenna optimization problem that offers information of the desired antenna currents for optimal radiation. The approach is powerful as many antenna problems can be formulated as convex optimization problems and are hence solved efficiently. A fundamental step in the formulation is to express the stored energy $[6,7]$ in the current density [4]. There are several unresolved question for the interpretation and accuracy of the stored energies $[3,6,7,11-14]$.

\section{Acknowledgments}

The support from the Swedish Foundation for Strategic Research and the Swedish Research Council is gratefully acknowledged.

\section{References}

[1] Y. Rahmat-Samii and E. Michielssen, Electromagnetic Optimization by Genetic Algorithms, 
ser. Wiley Series in Microwave and Optical Engineering. John Wiley \& Sons, 1999.

[2] R. L. Haupt and D. H. Werner, Genetic Algorithms in Electromagnetics. Wiley-IEEE Press, 2007.

[3] M. Gustafsson, M. Cismasu, and B. L. G. Jonsson, "Physical bounds and optimal currents on antennas," IEEE Trans. Antennas Propagat., vol. 60, no. 6, pp. 2672-2681, 2012.

[4] M. Gustafsson and S. Nordebo, "Optimal antenna currents for Q, superdirectivity, and radiation patterns using convex optimization," IEEE Trans. Antennas Propagat., vol. 61, no. 3, pp. 1109-1118, 2013.

[5] S. P. Boyd and L. Vandenberghe, Convex optimization. Cambridge Univ Pr, 2004.

[6] G. A. E. Vandenbosch, "Reactive energies, impedance, and $\mathrm{Q}$ factor of radiating structures," IEEE Trans. Antennas Propagat., vol. 58, no. 4, pp. 1112-1127, 2010.

[7] M. Gustafsson and B. L. G. Jonsson, "Stored electromagnetic energy and antenna Q," Lund University, Department of Electrical and Information Technology, P.O. Box 118, S-221 00 Lund, Sweden, Tech. Rep. LUTEDX/(TEAT-7222)/1-25/(2012), 2012, http://www.eit.lth.se.

[8] M. Cismasu and M. Gustafsson, "Antenna bandwidth optimization with single frequency simulation," IEEE Trans. Antennas Propagat., vol. 62, no. 3, pp. 1304-1311, 2014.

[9] —, "Multiband antenna Q optimization using stored energy expressions," IEEE Antennas and Wireless Propagation Letters, vol. 13, pp. 646-, 2014.

[10] M. Grant and S. Boyd, "CVX: Matlab software for disciplined convex programming, version 1.21," cvxr.com/cvx, Apr. 2011.

[11] W. Geyi, "On stored energies and radiation Q," arXiv preprint arXiv:1403.3129, 2014.

[12] M. Capek, L. Jelinek, P. Hazdra, and J. Eichler, "The measurable Q factor and observable energies of radiating structures," arXiv preprint arXiv:1309.6122, 2013.

[13] A. D. Yaghjian, M. Gustafsson, and B. L. G. Jonsson, "Minimum Q for lossy and lossless electrically small dipole antennas," Progress In Electromagnetics Research, vol. 143, pp. 641673, 2013.
[14] G. A. E. Vandenbosch, "Radiators in time domain, part II: finite pulses, sinusoidal regime and Q factor," IEEE Trans. Antennas Propagat., vol. 61, no. 8, pp. 4004-4012, 2013. 